\title{
Efecto de las capacitaciones en la reflexión sobre ética y
} corrupción

\author{
Cristian Pliscoff-Varas 1 \\ Nicolás Lagos-Machuca ${ }^{2}$ \\ 1 Universidad de Chile / Instituto de Asuntos Públicos, Santiago - Chile \\ 2 Contraloría General de la República de Chile, Santiago - Chile
}

El desafío de controlar la corrupción ha hecho evidente la necesidad de que las organizaciones gestionen la ética, desplegando instrumentos para reforzar los marcos éticos de los servidores públicos. Entre los instrumentos más usados para logar este objetivo, están las capacitaciones en ética, que proporcionarían elementos para que un funcionario enfrente de mejor forma una situación o un dilema ético. Pero, ¿es así?, ¿cuán eficientes son las capacitaciones en ética para los funcionarios públicos?, ¿tienen el mismo efecto entre los distintos tipos de funcionarios que forman parte del sector público? Para resolver estas cuestiones, en la presente investigación se presentan los resultados de la aplicación de un cuestionario previo y posterior al desarrollo de una capacitación en ética para funcionarios que se desempeñan en la administración central y local en Chile. Los resultados indican de manera exploratoria que luego de la capacitación existe una adecuación de los participantes a los marcos éticos, identificando un efecto positivo diferenciado en mujeres y personas de reciente ingreso a la administración pública. Se requieren más investigaciones para perfeccionar el instrumento y así aportar de mejor forma a una administración pública más proba. El texto finaliza con algunas propuestas de mejora a este tipo de intervenciones.

Palabras clave: ética pública; integridad; corrupción; capacitaciones; Chile.

\section{Efeito das capacitações na reflexão sobre ética e corrupção}

O desafio de controlar a corrupção deixou clara a necessidade das organizações fazerem uma gestão da ética, implantando instrumentos que reforcem os marcos éticos dos servidores públicos. Entre os instrumentos mais utilizados para atingir esse objetivo está o treinamento em ética, que forneceria elementos para que um funcionário possa enfrentar melhor uma situação ou dilema ético. Mas é assim? Quão eficiente é o treinamento em ética para funcionários públicos? Eles têm o mesmo efeito entre os diferentes tipos de funcionários que fazem parte do setor público? Para resolver essas questões, esta pesquisa apresenta os resultados da aplicação de um questionário antes e depois do desenvolvimento de um treinamento em ética para funcionários que atuam na administração central e local no Chile. Os resultados indicam de forma exploratória que após a formação ocorre uma adequação dos participantes aos referenciais éticos, identificando um efeito positivo diferenciado em mulheres e pessoas que ingressaram recentemente na administração pública. Mais pesquisas são necessárias para aperfeiçoar o instrumento e, assim, contribuir melhor para uma administração pública mais proba. O texto termina com algumas propostas de aprimoramento para este tipo de intervenção.

Palavras-chave: ética pública; integridade; corrupção; capacitações; Chile.

\section{Effect of training on reflection about ethics and corruption}

The challenge of curbing corruption requires public organizations to implement ethics management, deploying a myriad of instruments to reinforce ethical frameworks of public employees. Ethical training is among the most used instruments to achieve this goal, which should provide elements to public officials to face ethical dilemmas effectively. Yet, is that the case? How efficient are ethical training efforts for public servants? Do they have the same effect among different types of employees? In order to answer these questions, this article shows the results of pre and post-test questionnaires applied to participants of ethics training workshops for employees in the central and local government in Chile. The exploratory findings show that after the workshops, it is possible to see an improvement in the ethical frameworks among participants, showing a positive effect for women and those who recently started working in the public sector. More research is required to improve the instrument and strengthen public integrity. The article concludes with proposals to improve these kinds of training activities.

Keywords: public ethics; integrity; corruption; training; Chile. 


\section{INTRODUCCIÓN}

¿Las capacitaciones sobre ética, tienen el mismo efecto para los distintos tipos de funcionarios que se exponen a estas actividades? Esta pregunta aborda dos temáticas de gran relevancia para los estudios de ética pública y corrupción. Primero, que las organizaciones deben gestionar la ética (ethics management), de forma de generar un mejor estándar de comportamiento de quienes trabajan en las instituciones públicas (Menzel, 2001, 2007). Segundo, que las y los funcionarios públicos, pueden ser capacitados en ética pública y, por lo tanto, pueden estar mejor preparados para enfrentarse a situaciones donde se ponen en tela de juicio los principios y valores que observan un actuar determinado. En este documento nos referiremos en particular a la ética de las y los funcionarios públicos, diferenciándola de la ética pública que deberían observar autoridades políticas o similares. Entendemos la relación entre ambas, pero en este documento nos centraremos en la ética que deben observar las y los funcionarios públicos en particular.

La literatura muestra que existen diferentes modos y efectos de las capacitaciones en ética para las y los funcionarios del sector público. Siguiendo con el modo tradicional de enfrentar el tema de la ética en general en América Latina, la capacitación en ética pública tiende a ser más bien orientada a la información de materias éticas, siguiendo un enfoque de la obediencia, por sobre uno de la integridad. Si bien el tema de las capacitaciones en ética tiene una larga data de reflexión, aún existen materias que no han sido adecuadamente tratadas. Especial mención se debe hacer respecto de si las capacitaciones tienen o no el mismo efecto entre diferentes grupos de funcionarios o funcionarias. Este tema reviste especial relevancia puesto que, en caso de haber diferentes efectos, sería necesario diseñar instrumentos diferenciados por tipos de funcionarios, para alcanzar un impacto mayor. El presente artículo pretende aportar en ese ámbito de la reflexión académica, del cual existe evidencia que ha sido poco estudiado (Cochrane, 2020; Toro \& Rodríguez, 2017).

En este estudio exploratorio, buscamos responder la pregunta ¿cuál es el efecto que tienen las capacitaciones en ética entre los diferentes tipos de funcionarios públicos? Para evidenciar este efecto compararemos los resultados que obtuvimos de la aplicación de un test antes y después de la capacitación. El instrumento contiene un grupo de comportamientos que evidencian situaciones cotidianas a las cuales se podrían enfrentar los funcionarios públicos. Se le pide a cada participante que señale en una escala likert cuán reprochables y/o sancionables le parece cada una de las acciones que se describen. Desde un punto de vista práctico, este trabajo entrega reflexiones que permiten destacar el aporte que las capacitaciones éticas han tenido en los funcionarios capacitados. Desde un punto de vista teórico, el presente trabajo aporta en reconocer las diferencias que existen entre grupos de funcionarios y funcionarias, al momento de ser objetos de actividades formativas con un modelo de capacitación enfocado en la integridad. En particular, se destaca el efecto diferenciado entre hombres y mujeres, y las y los empleados con menos experiencia laboral. Una vez presentada la literatura relevante y el contexto donde se realiza la investigación, se presenta el diseño de la aplicación de la encuesta y los resultados de la misma. Se finaliza el artículo con aportes prácticos y teóricos sobre la materia. 


\section{REVISIÓN DE LA LITERATURA}

Los gobiernos han abordado el problema de la ética pública a partir de dos enfoques (Huberts \& Hoekstra, 2016; Jiménez Asensio, 2017; Maesschalck, 2004; Organisation for Economic Co-operation and Development [OECD], 2020). Por un lado, está el llamado "enfoque de la obediencia" (compliance approach), que ha puesto especial énfasis en una perspectiva normativa sobre la ética, centrando en lo que prescriben los cuerpos legales a las conductas esperadas, también conocido como el "low road" (Rohr, 1989). Es un enfoque que se ha basado en la supervisión y el control por sobre otras consideraciones. En cambio, el "enfoque de la integridad" (integrity approach) pone énfasis en los valores y responsabilidades que las personas deben tener en cuenta al momento de actuar como funcionario público (Lewis \& Gilman, 2012, p. 14). Para reforzar el comportamiento ético de las y los funcionarios no es adecuado centrarse en los controles y castigos, sino en los valores y principios que cada individuo observa. Como lo señala Roberts (2009) la estrategia dominante ha sido intervenir éticamente a las organizaciones desde una perspectiva de la obediencia. Las principales formas de intervención han sido los cambios organizacionales, las destituciones u otros modos de sanción por las conductas reñidas con la probidad. Este tipo de intervenciones se hacen insuficientes toda vez que no generan las condiciones para que en el futuro, las y los funcionarios públicos se enfrenten adecuadamente a potenciales dilemas éticos.

Esta mayor complejidad para abordar el problema de la ética y la corrupción, ha llevado a pensar el problema de las intervenciones organizacionales en la materia, de una forma más omnicomprensiva. En particular, podemos ver este enfoque en lo que Kaptein (2009) llama los "programa de ética", que son formas de control organizacional formal para crear una cultura ética, e impedir conductas desviadas o corrupción. La efectividad de este tipo de acciones ha sido documentada en diversos estudios (Beeri, Dayan, Vigoda-Gadot \& Werner, 2013; Kaptein, 2015; Osorio \& Rodríguez, 2018; Park \& Blenkinsopp, 2013; Yizraeli \& Shilo, 2001), donde se demuestra que tanto en el sector público como privado se pueden y deben instalar estos componentes formales, de forma de reforzar la ética de la organización y sus funcionarios. Dentro de los elementos que se incorporan en estos programas de ética están los códigos de ética, el liderazgo ético, el clima ético, los encargados de ética, los sistemas de denuncia, entre otros (Kaptein, 2009). Junto con estos instrumentos se reconoce la necesidad de incluir las capacitaciones para lograr los objetivos de los programas.

Como todo proceso formativo, las capacitaciones en ética son útiles, porque no solo permiten entregar o actualizar información sobre la materia, sino que también crean un espacio adecuado donde se reflexione sobre situaciones particulares que se dan en el trabajo regular en las organizaciones (Frisque \& Kolb, 2008), ya que no siempre quienes trabajan en una entidad, poseen la sofisticación cognitiva, o alfabetización moral como señala Tuana (2007), o incluso el coraje para enfrentar situaciones complejas en este ámbito (Trevino \& Brown, 2004). Sin embargo, la literatura es poco clara respecto de cuán efectivas son estas capacitaciones para reforzar significativamente el comportamiento ético de los distintos grupos de funcionarios públicos. En cierta medida, se asume que toda capacitación en ética tiene un impacto entre quienes participan en estas actividades. Van Montfort, Beck y Twijnstra (2013) señala que la literatura en las ciencias sociales sobre el particular es muy limitada. Esta incertidumbre no es monopolio del sector público, de hecho la temática ya ha estado presente en la literatura de los negocios desde hace ya un tiempo (Delaney \& Sockell, 1992; Thompson, 1990), donde se señalaba que si bien el tema es relevante, en las propias empresas la 
capacitación era limitada o inexistente, o no está claro cuán efectivas son. Últimamente, la temática ha recobrado interés (Steele et al., 2016), en gran parte por la tendencia global de mayores estándares éticos en las empresas.

En el sector público, la reflexión sobre esta materia ha estado presente de forma más limitada (Cochrane, 2020; Pallai \& Gregor, 2016). De hecho, en una de la publicaciones más relevantes de esta área de reflexión académica, la revista Public Integrity, hay varios textos sobre la materia, sin embargo, en un número muy limitado respecto de otras áreas de la reflexión académica (Menzel, 2015). En el caso de otros journals importantes en el ámbito disciplinario de la administración pública, la producción académica también es muy limitada, destacando textos más bien descriptivos (Dwivedi \& Engelbert, 1981; Worthley \& Grumet, 1983) o propositivos (Worthley, 1981). En el caso de América Latina, en las principales revistas del área, tal como se presenta en Pliscoff (2019), la producción académica sobre ética en el servicio público es limitada, y más aún en capacitación sobre el tema. El texto de Van Montfort et al. (2013) es sin duda el más relevante para la temática que abordamos en este artículo.

Contrario a la limitada generación de conocimiento sobre la materia, la OECD (2020) ha evidenciado que en la actualidad los gobiernos implementan capacitaciones éticas regularmente. Desde un punto de vista teórico, estos esfuerzos adquieren diferentes formas. Como lo señala Hejka-Ekins (2001), se pueden diferenciar los focos, rasgos, resultados, contenidos y métodos de aprendizaje que van a variar de acuerdo al modelo de control ético adoptado, como se puede apreciar en el Cuadro 1.

\section{CUADRO 1 TIPOLOGÍA DE MODELOS DE CAPACITACIÓN EN ÉTICA EN EL SECTOR PÚBLICO}

\begin{tabular}{lcc} 
Factor & Obediencia & Integridad \\
\hline Foco & Individual & Individual \\
Rasgo & Legal & Normativo \\
Resultado & Comportamiento legalmente correcto & Conducta éticamente correcta \\
Contenido & Leyes sobre ética, reglas y regulaciones & Estándares éticos y procesos de toma de decisiones \\
Método de aprendizaje & Pedagógico & Andragógico
\end{tabular}

Fuente: Adaptado de Hejka-Ekins (2001, p. 89).

A partir de esta tipología, se comprende que las capacitaciones pueden representar un aporte a las organizaciones por dos vías. En primer lugar, a través de la definición y socialización de normas y reglas que orienten el comportamiento ético (Hejka-Ekins, 2001, p. 83) y, en segundo lugar, mediante el fortalecimiento de la capacidad reflexiva de los miembros de la organización (Cooper, 2006; Gomes, 2014). Esta capacidad reflexiva permite tanto la detección de los problemas éticos como las formas en que se pueden abordar (Tuana, 2007). En este sentido, el efecto ideal que una capacitación en ética debería ser el aumentar el conocimiento y manejo de las normas sobre ética, y el incremento en la capacidad para distinguir las conductas aceptadas de las que no lo son. 
Cochrane (2020) plantea que uno de los problemas para poder determinar si las capacitaciones en ética son o no pertinentes, se debe a la falta de instrumentos adecuados para dar cuenta del efecto que estas acciones tienen. Pero incluso, si se mejoraran las técnicas de evaluación, dando cuenta de técnicas adecuadas para objetivos buscados (D. Kirkpatrick \& J. Kirkpatrick, 2006), aún quedan algunos temas poco claros, que influyen en el efecto de estos esfuerzos organizacionales. Por ejemplo, permanecen algunas dudas respecto de cómo el efecto de las capacitaciones pudiese diferir entre los distintos tipos de funcionarios existentes en una organización. Medeiros et al. (2017) plantean que el efecto de las capacitaciones difiere entre estudiantes de pregrado y estudiantes de posgrado o con experiencia profesional, pero en el ámbito de los negocios y sin distinguir entre personas con mayor o menor experiencia profesional. Swamy et al. (2001) por otro lado plantean que las mujeres tienden a tener una actitud de mayor rechazo a las prácticas contrarias a la ética, lo cual podría tener un efecto en el impacto de las capacitaciones en materias de ética (Ritter, 2006).

En el presente artículo, trataremos de aportar a esta discusión, a partir del análisis de los resultados antes y después de capacitaciones en ética. Por ello, la originalidad del trabajo reside en analizar el efecto de las capacitaciones sobre ética pública en un contexto profesional como el chileno, el cual ha sido poco estudiado. Pero además, pretende aportar en la discusión teórica sobre en qué grupos de personas tienen un mayor efecto las capacitaciones con énfasis en la integridad.

\section{CONTEXTO DE LA INVESTIGACIÓN}

Desde 2017, el Centro de Estudios de la Administración del Estado (CEA), dependiente de la Contraloría General de la República de Chile (CGR), imparte el curso "Inducción a la Administración del Estado" cuyo objetivo es perfeccionar los conocimientos, habilidades y valores de las personas de reciente ingreso a la Administración del Estado a nivel central y municipal.

El módulo final de este curso, referido a Ética Pública, Probidad y Transparencia, busca que los y las participantes conozcan el sentido y alcance de la ética, la probidad y la transparencia como herramientas de lucha contra la corrupción. Durante las cuatro horas de duración del bloque se presentan tres conceptos con un alcance teórico y normativo; se conceptualiza el fenómeno de la corrupción y se finaliza reflexionando sobre el rol que desempeñan los funcionarios públicos en el fortalecimiento de la probidad administrativa.

El módulo es impartido por relatores de la CGR a grupos de entre 10 y 35 funcionarios como máximo. Durante la actividad, los relatores a cargo de la actividad combinan instancias expositivas con elementos participativos donde se promueve la reflexión de las personas en situaciones habituales que servidores y servidoras públicas enfrentan.

Para cumplir con el objetivo señalado y a partir de la tipología señalada en el apartado anterior, se ha optado por una metodología enfocada en el fortalecimiento de la integridad de los participantes. En este sentido, la actividad hace énfasis en aquellas conductas socialmente aceptadas en el ámbito público y la relevancia de elevar los estándares éticos en los procesos de toma de decisiones. Siguiendo la clasificación propuesta por Medeiros et al. (2017) este curso tiene los siguientes atributos: 


\section{CUADRO 2 CLASIFICACIÓN DEL TIPO DE ACTIVIDAD}

\begin{tabular}{ll} 
Atributo & \multicolumn{1}{c}{ Caso del curso CEA CGR } \\
$\begin{array}{ll}\text { 1. Categoría } & \text { Sensibilización ética. } \\
\text { 2. Mecanismo de evaluación de efectividad } & \text { Grupo único con evaluación pre y post. } \\
\text { 3. Propósito } & \text { Formación profesional con participación obligatoria. } \\
\text { 4. Contenidos } & \text { Revisión acotada de contenidos (normas, códigos de ética, comportamientos éticos). } \\
\text { 5. Método de enseñanza } & \text { Utilización acotada de metodologías (resolución grupal de problemas). } \\
\text { 6. Procesos de análisis } & \text { Limitado (concientización ética grupal). }\end{array}$
\end{tabular}

Fuente: Elaborado por los autores.

\section{DISEÑO METODOLÓGICO}

Con el objetivo de identificar el impacto diferenciado que esta actividad tiene en los distintos grupos de participantes, utilizaremos una técnica pre-post, donde compararemos los resultados del grupo capacitado, antes y después de la actividad. Así, se pretende identificar diferencias entre los tipos de personas mediante un análisis que considere las variables de control asociadas al género, edad y años en la administración pública. Creemos que esta metodología analizada por Steele et al. (2016) y aplicada anteriormente en estudios similares del sector público (Van Montfort et al., 2013) y privado (Ritter, 2006) nos permite verificar en particular el efecto a corto plazo de esta capacitación, toda vez que se aplicó el instrumento antes de iniciar la capacitación y luego de ella.

En relación al instrumento para detectar el efecto de la capacitación en ética entre las y los funcionarios, optamos por crear un instrumento donde se incluyeron un listado de 15 situaciones a las cuales cualquier persona que se desempeñe en una institución pública podría verse enfrentado durante su trabajo diario. Esta decisión de diseño de la investigación, se basa en lo que Neu et al. (2015), plantean, en términos que las normas y códigos son importantes, pero lo esencial en la reflexión sobre ética, son las prácticas diarias. Para abordar distintos tipos de acciones que podrían vulnerar el correcto comportamiento de un servidor público y con la finalidad de facilitar el posterior análisis de las situaciones, el cuestionario contempló tres tipos presentados sin algún orden en particular ${ }^{1}$. Así tenemos tres tipos de situaciones asociadas a distintas formas o tipos de faltas éticas:

1. Grupo 1: acciones y/o situaciones en las cuales el individuo estaría transgrediendo una norma legal del ámbito administrativo o, incluso, penal. Se refieren a aquellas formas más clásicas y evidentes de corrupción que, en ciertos casos, implican un beneficio monetario directo. En este sentido, debiesen ser más fáciles de identificar como situaciones indeseables. En este grupo encontramos acciones de soborno, cohecho, revelación de secreto profesional y prevaricación administrativa.

${ }^{1}$ Esta agrupación es a partir de la clasificación de tipos de corrupción propuesta por Bermúdez (2017). 
2. Grupo 2: situaciones que podrían vulnerar algún tipo de norma administrativa o penal, sin embargo, se refiere a figuras menos evidentes que el soborno o el cohecho. Se incluyen formas más sofisticadas de corrupción donde, en general, no es el dinero el beneficio directo que obtiene el individuo involucrado. Por esto, se considera que podrían ser situaciones más difíciles de identificar como prácticas incorrectas. Se encuentran aquí faltas asociadas a los conflictos de intereses, la negociación incompatible o el tráfico de influencias.

3. Grupo 3: incluye acciones que no involucran necesariamente figuras penales o administrativas claras. Por el contrario, se trata de situaciones indeseables ya que afectan el comportamiento íntegro que el personal público. A partir de esto, se considera que serían prácticas que se encuentran en una zona gris donde no existe total conciencia de su posible vinculación con acciones de corrupción. En general se incorporan acciones como el mal uso de recursos públicos para fines personales o malas prácticas administrativas.

A continuación, se detallan las situaciones éticas clasificadas según esta distinción metodológica:

\section{CUADRO 3 CLASIFICACIÓN DE ACCIONES SEGÚN GRUPO DE SITUACIONES}

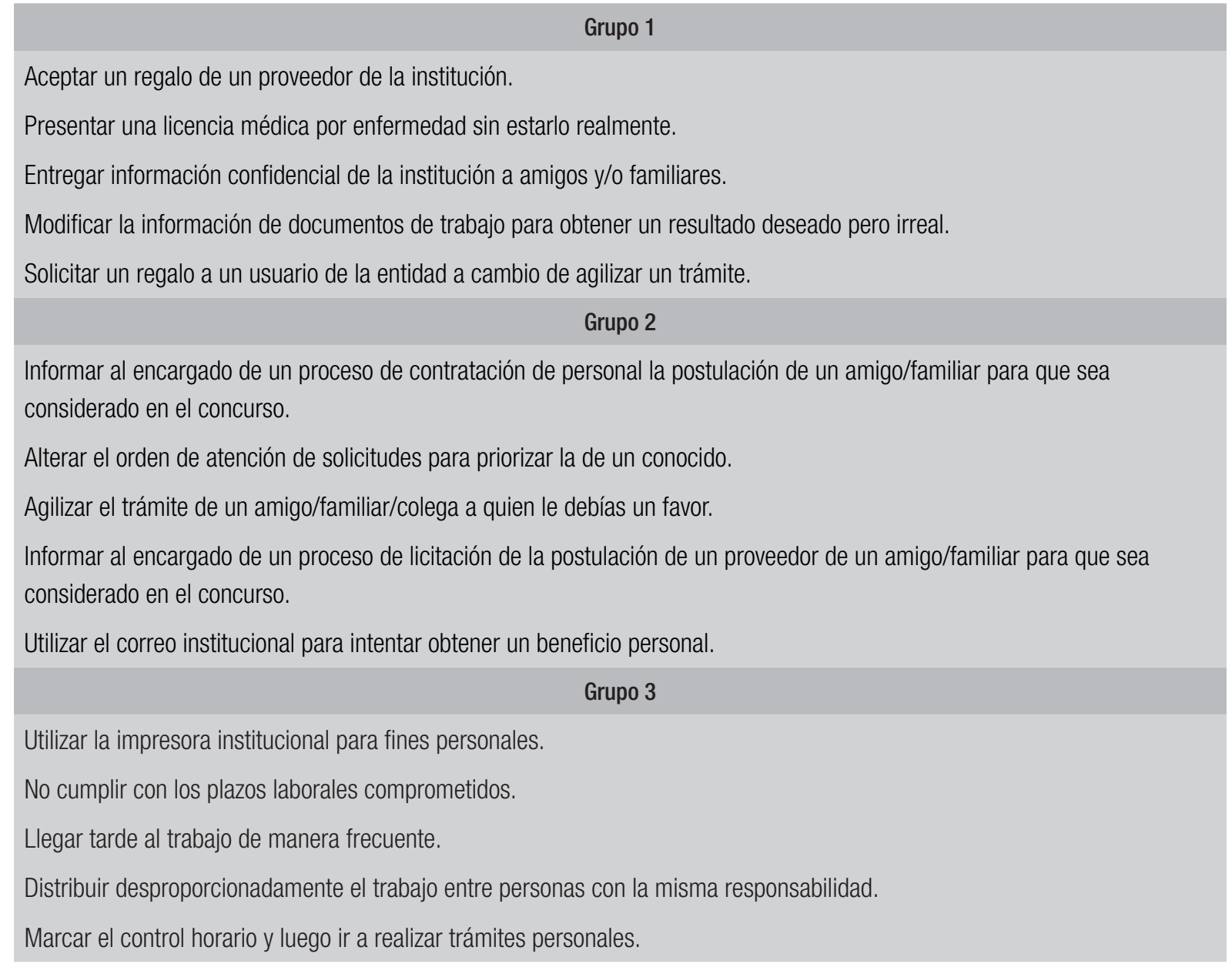

Fuente: Elaborado por los autores. 
Así, se le solicitó a cada participante que evaluara en una escala de 1 a 9 cuán reprochables eran esas situaciones, donde 1 era "Irreprochable o admisible" y 9 era "Totalmente reprochable, debiese tener una sanción penal". El resultado esperado, donde se hace evidente que la capacitación cumple el efecto, radicaría en que al finalizar la jornada las y los participantes señalen como éticamente reprochables o inaceptables las acciones que se someten a evaluación. Esto debiese verse traducido en un aumento en la calificación que las personas realizan a la gravedad de las situaciones señaladas.

El cuestionario fue aplicado en papel al iniciar y al finalizar la capacitación. Para poner en contexto a los participantes, se informó a las personas que desempeñaron la función de relatoría del curso sobre el objetivo del estudio y se les pidió que explicaran que el único fin del instrumento era evaluar y perfeccionar el impacto que la capacitación tenía en las y los participantes. De igual forma, se les alentó a explicar que si bien la encuesta era anónima era muy relevante que completaran la caracterización, donde se solicita señalan: género, edad, y años trabajando en la administración pública. El instrumento fue aplicado en las clases desarrolladas los meses de abril y octubre de 2019 por los relatores o relatoras de los cursos y luego centralizadas por los autores de este estudio quienes procesaron y analizaron los resultados obtenidos.

\section{ANÁLISIS DE RESULTADOS}

Luego de realizadas todas las intervenciones planificadas se logró obtener un total de 170 encuestas; 89 correspondían a respuestas antes de la capacitación y 81 a cuestionarios luego de la capacitación. Los resultados generales se presentan en los siguientes gráficos.

\section{GRÁFICO 1 CARACTERIZACIÓN DE PARTICIPANTES}

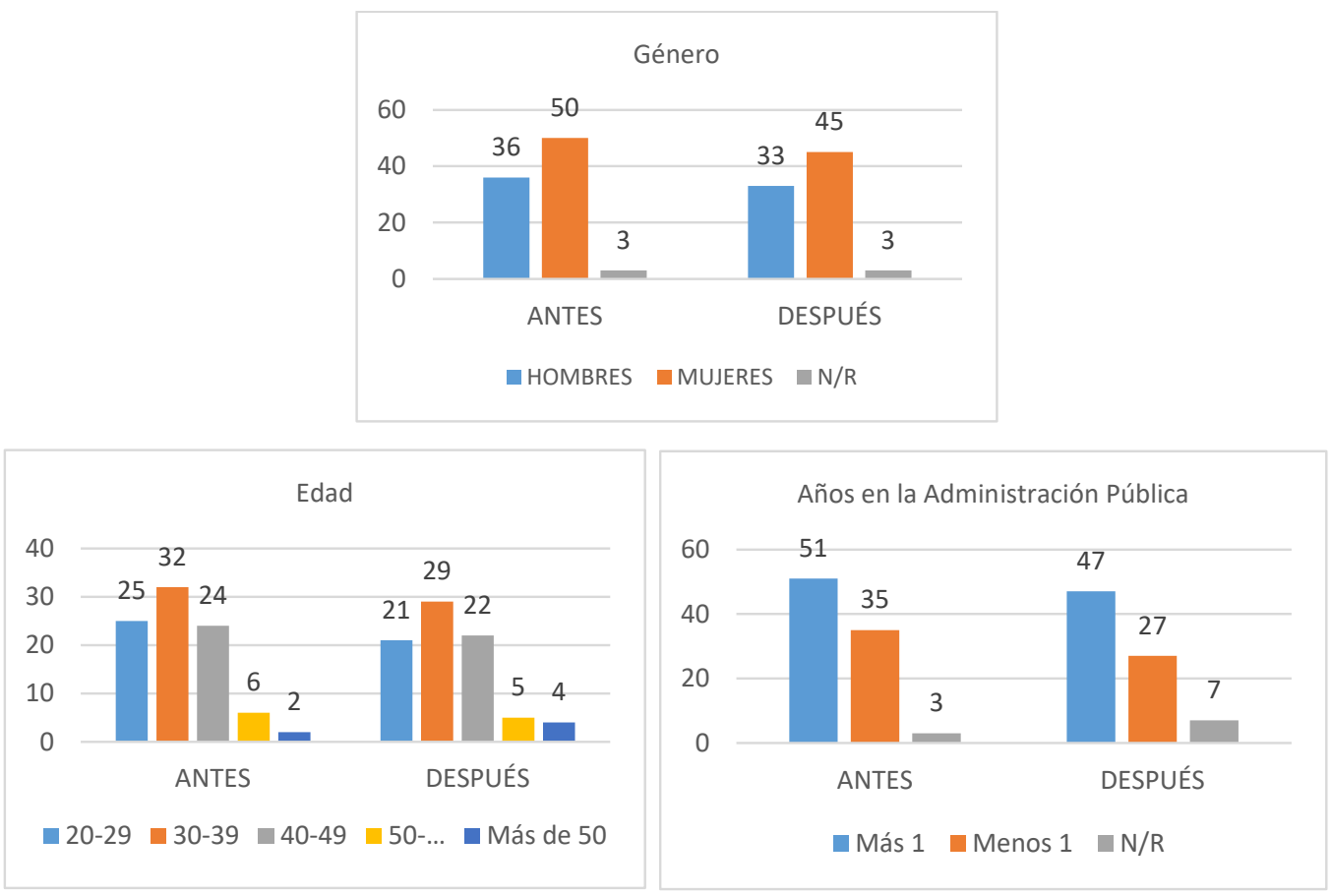

Fuente: Elaborado por los autores. 
El grupo participante está conformado principalmente por mujeres con una edad de entre $30 \mathrm{y}$ 39 años y trabajando hace más de un año en la Administración Pública, siendo la edad media de los participantes de 35,8 años y el promedio de años trabajando en la administración pública de 4,9 años. Finalmente se destaca que las distribuciones entre las características de los participantes se mantienen similares antes y después de la intervención. Los estadísticos descriptivos de cada dimensión pueden ser revisados en el Anexo (Tabla 1). La fiabilidad del instrumento fue evaluada separadamente, obteniendo resultados satisfactorios; con un Alfa de Crombach de 0.895 para antes de la capacitación y 0.914 para después. En el Anexo (Tabla 2) se pueden observar los resultados para cada situación evaluada pre y post capacitación.

Como primer punto a destacar en el análisis de los resultados, podemos señalar que hubo un aumento en la percepción ética en todas las situaciones presentadas. Mientras el promedio global del puntaje asignado a las acciones antes de la capacitación es 6.65, luego de la actividad esta calificación general alcanza un 7.07. Esta alza podría evidenciar que la capacitación logró modificar la forma en que las personas se enfrentaban y calificaban la gravedad de las situaciones presentadas. Siguiendo a Tuana (2007), se cumpliría el sentido de este tipo de capacitaciones, puesto que este cambio evidenciaría un desarrollo de habilidades de razonamiento ético, estimulando la reflexión ética, que facilita la identificación de situaciones que podrían afectar el comportamiento probo de los miembros de la institución.

Luego, al controlar por los tres grupos de situaciones que el cuestionario señalaba, es posible identificar que las puntuaciones tienen modificaciones diferentes según el tipo de acción. Mientras los casos correspondientes al tercer nivel de corrupción tienen un aumento de 0.61 puntos (de 5.96 a 6.57); las situaciones correspondientes al segundo nivel de corrupción se elevan en 0.41 puntos (de 6.67 a 7.08 ) y; las acciones del primer nivel se incrementan solo en 0.24 puntos (de 7.34 a 7.58). Estos cambios harían suponer que la capacitación logra modificar la percepción ética de forma diferenciada según el tipo de acción al que la persona de enfrenta.

En el siguiente cuadro se señalan aquellas acciones que presentan mayores puntajes de variación comparando antes y después de la capacitación.

\section{CUADRO 4 ACCIONES CON MAYOR DIFERENCIA PRE Y POST CAPACITACIÓN}

\begin{tabular}{l|ccc}
\hline Acción & Pre & Post & Diferencia \\
\hline $\begin{array}{l}\text { i) Informar al encargado de un proceso de contratación de personal la } \\
\text { postulación de un amigo/familiar para que sea considerado en el concurso. }\end{array}$ & 5.90 & 6.74 & 0.84 \\
\hline ii) Marcar el control horario y luego ir a realizar trámites personales. & 6.63 & 7.47 & 0.84 \\
\hline iii) No cumplir con los plazos laborales comprometidos. & 5.96 & 6.77 & 0.81 \\
\hline iv) Llegar tarde al trabajo de manera frecuente. & 6.07 & 6.63 & 0.53 \\
\hline
\end{tabular}

Fuente: Elaborado por los autores. 
Las mayores variaciones no se encuentran en aquellas situaciones más complejas, de mayor impacto financiero o con una relación evidente con casos de corrupción. Por el contrario, los principales cambios se encuentran en aquellos asuntos que hacen referencia a situaciones en apariencia sencillas, de bajo impacto para el funcionamiento de la institución o sin un daño directo a los recursos públicos. Esto resulta del todo llamativo, puesto que la intervención parece facilitar que las personas identifiquen el impacto que la ocurrencia de estas acciones genera en el clima ético al interior de la organización, más allá del daño patrimonial o reputacional que estas situaciones podrían implicar. Como bien lo señalan Park y Blenkinsopp (2013), las intervenciones en ética están condicionadas por la cultura ética de las organizaciones. Creemos que este tipo de capacitaciones que ocurren en un espacio diferente al institucional, permiten "sacar" al funcionario de la cultura ética de la organización, y les permite reconocer que algunas prácticas aceptadas en la organización, aunque aparentemente triviales, no serían probas.

Esto viene a reforzar la idea que el cambio en el enfoque de las intervenciones en ética desde uno centrado en la obediencia a un centrado en la integridad (Hejka-Ekins, 2001), permite que los funcionarios puedan reconocer que existen prácticas que pueden ser legalmente aceptadas, pero no necesariamente éticamente justificables.

Lo anterior se refuerza al enlistar aquellas afirmaciones opuestas, es decir, con una menor variabilidad luego de la intervención, permite identificar:

\section{CUADRO 5 ACCIONES CON MENOR DIFERENCIA PRE Y POST CAPACITACIÓN}

\begin{tabular}{|c|c|c|c|}
\hline Acción & Antes & Después & Diferencia \\
\hline $\begin{array}{l}\text { i) Solicitar un regalo a un usuario de la entidad a cambio de agilizar un } \\
\text { trámite. }\end{array}$ & 7.78 & 7.78 & - \\
\hline $\begin{array}{l}\text { ii) Informar al encargado de un proceso de licitación la postulación de } \\
\text { un proveedor de un amigo/familiar para que sea considerado en el } \\
\text { concurso. }\end{array}$ & 7.29 & 7.30 & 0.01 \\
\hline iii) Aceptar un regalo de un proveedor de la institución. & 6.44 & 6.51 & 0.06 \\
\hline
\end{tabular}

Fuente: Elaborado por los autores.

Estas acciones tienen dos elementos en común. El primero de ellos es que todas las situaciones hacen referencia a acciones que podrían ser relacionadas con las figuras penales que clásicamente se consideran como corrupción, es decir; cohecho y tráfico de influencias. El segundo elemento, asociado a los casos (ii) y (iii), guarda relación con que ambos se refieren a la misma área: compras públicas. Estas materias se encuentran ampliamente reguladas en el país. En la práctica, esto se ha traducido en un alto grado de conocimiento por parte del personal público de las normas, prohibiciones y buenas prácticas que involucran las compras públicas (Inter-American Development Bank, [IDB] 2008; Dirección Chile Compra, 2018; Lara A., 2017; Meyer \& Fath Meyer, 2012). A partir de lo anterior, es posible establecer que este tipo de capacitaciones tendría un efecto menos evidente en situaciones en que se contraviene una acción regulada directamente a través de una norma. Es decir, en acciones 
donde la corrupción es más evidente como resultado de una regulación clara (como en las figuras penales) o debido a un amplio conocimiento de las normas (como en compras públicas), este tipo de intervenciones tiende a tener un menor efecto.

Por otro lado, se analizaron aquellos casos que tuvieron mayores modificaciones luego de la capacitación. Bajo este criterio, se destacan dos variables: "Informar el encargado de un proceso de contratación de personal la postulación de un amigo/familiar para que sea considerado en el concurso" y "Marcar el control horario y luego ir a realizar trámites personales", ambos con un aumento en la calificación recibida de 0,84 puntos. El primero de estos, recibe una calificación inicial de 5,90 y luego una evaluación de 6,74 puntos y al realizar un análisis de los resultados se observa que la variación está explicada por el comportamiento específico de mujeres participantes, lo que será explicado más adelante.

De igual forma, el segundo caso avanza de 6.63 a 7.47, y - a diferencia del anterior- los datos no arrojan relaciones relevantes o resultados asociados a algún grupo en particular que haga mover la media de puntuación. La respuesta se puede encontrar si analizamos la estructura de la capacitación y la manera en que esta situación se aborda durante la jornada. Considerando lo señalado en el Anexo (Tabla 1) "Estructura del módulo", mientras la mayoría de los casos son abordados de manera general el caso de aquellas personas que registran su ingreso en el control horario y luego realizan actividades con fines personales se aborda de una manera diferenciada. A continuación, se presenta en detalle la metodología utilizada:

\section{CUADRO 6 METODOLOGÍA UTILIZADA EN EL CASO DE MALAS PRÁCTICAS EN EL CONTROL HORARIO}

\begin{tabular}{|c|c|c|}
\hline $\mathrm{N}^{\circ}$ & Objetivo & Descripción \\
\hline 1 & Enmarcar & $\begin{array}{l}\text { Reflexión grupal sobre la relevancia de las malas prácticas administrativas como tercer nivel de } \\
\text { corrupción. }\end{array}$ \\
\hline 2 & Aplicar & $\begin{array}{l}\text { Reflexión grupal sobre el impacto que tiene en el Estado la suma agregada de las malas prácticas que } \\
\text { a diario se realizan. }\end{array}$ \\
\hline 3 & Vincular & $\begin{array}{l}\text { Reflexión grupal sobre aquellas situaciones habituales que podrían ser catalogadas como malas } \\
\text { prácticas, promoviendo que se mencione el hecho de marcar el control horario y luego ir a hacer } \\
\text { actividades personales. }\end{array}$ \\
\hline 4 & Ejemplificar & Reflexión grupal sobre implicancias de esta mala práctica, señalando el lineamiento que la CGR definió. \\
\hline
\end{tabular}

Fuente: Elaborado por los autores.

La estructura metodológica utilizada para abordar esta temática no fue replicada en otros casos y sería el factor específico que permitiría comprender la variación existente en la percepción ética del grupo. En consecuencia, la realización de un análisis concreto, detallado y aplicado de ciertos casos que podrían violar la integridad organizacional modificaría la percepción ética de una manera más eficiente. En otras palabras, la focalización de las capacitaciones de integridad en determinadas situaciones éticas parece ser un elemento indispensable para asegurar la eficacia de estas actividades. 
Un segundo gran tema que surge del análisis de los resultados, guarda relación con identificar la presencia de posibles comportamientos similares entre los distintos grupos de participantes. Por ello, se procedió a analizar el coeficiente de correlación de Pearson entre las 15 situaciones éticas examinadas y las tres variables de control disponibles: género, edad y años de trabajo en la administración pública. Como se puede observar en el Anexo (Tabla 2) los resultados de este ejercicio no permiten identificar correlaciones significativas en ninguno de los cruces realizados. Sin perjuicio de esto, al realizar una revisión específica sobre las variaciones que se dan dentro de los distintos grupos, es interesante destacar los comportamientos que se evidencian en los y las participantes según sus años en la administración pública y según su género.

Al controlar por antigüedad en la Administración Pública aquellos casos asociados al primer y segundo grupo, presentan diferencias similares en los distintos participantes. Sin embargo, al revisar la variación del tercer grupo, es decir, aquellas acciones más cercanas a faltas éticas que a figuras penales, observamos que los participantes con más de un año de antigüedad modifican su percepción ética en menor medida (0.49) que aquellos que llevan menos de un año en el sector público (0.70). Lo anterior es especialmente evidente en el caso "No cumplir con los plazos laborales comprometidos" donde las personas de menos de un año en la Administración modifican levemente su percepción en comparación con el otro grupo de participantes: 0.56 y 1.12 respectivamente.

Este hallazgo es algo que no ha sido reconocido en la literatura previamente. Existe cierta relación con el trabajo realizado por Medeiros et al. (2017) quienes establecen que existe una diferencia en la capacitación o formación en ética entre estudiantes universitarios y profesionales efectuando sus MBA. Pero como ven, esos datos son con un público diferente. En nuestro caso, creemos que este efecto se explica porque las actitudes que no son delitos, pero que implican una falta ética, deben ser reconocidas como no apropiadas, a partir de situarse en la lógica y especificidad propia del sector público (Boyne, 2002), lo cual se va desarrollando con el tiempo. Del mismo modo, puede reflejar que estas materias, a quienes no han tenido experiencia en el sector público, no les asignan la relevancia que tienen, pero que luego de la capacitación, la reconocen.

En segundo lugar, al analizar el promedio general del comportamiento de la percepción ética según género, se identifica una diferencia relevante entre ambos grupos. Mientras las mujeres aumentan la calificación promedio en 0.73 puntos, los hombres disminuyen su evaluación media en 0.13 puntos. Esto se repite si se analiza según acciones del primer, segundo y tercer grupo de situaciones éticas. Los hombres tienen variaciones de $-0.27,-0.30$ y 0.20 y las mujeres $0.53,0.83$ y 0.85 respectivamente. Esto se observa con mayor claridad en el escenario: "Informar al encargado de un proceso de contratación de personal la postulación de un amigo/familiar para que sea considerado en el concurso" en que luego de la capacitación los hombres disminuyen 0.36 puntos y las mujeres aumentan su calificación en 1.63 puntos.

Los resultados que se han alcanzado son similares a estudios realizados anteriormente en estudiantes de administración (Ritter, 2006) en que se evidenció que hombres y mujeres tuvieron reacciones distintas luego de ser sometidos a clases de ética. Esta situación se explica, argumenta la autora, por el tipo de razonamiento moral al que mujeres recurren para resolver problemas éticos. Mientras los hombres tienden a resolver dilemas a través de comportamientos basados en reglas, las mujeres recurren a un análisis específico del contexto. Lo que haría suponer, en este caso, que durante la jornada las mujeres incorporan a su análisis de contexto aquellas normas jurídicas o reglas sociales que la capacitación busca entregar. Esta situación habría promovido que mujeres reflexionen 
desde una nueva vereda sobre los casos presentados, aumentando su visión crítica respecto de qué situaciones son éticamente aceptables y cuáles no. Pero junto con ello, se ha planteado que las mujeres tienden a caer en menor número que los hombres en actos de soborno (Swamy, Knack, Lee \& Azfar, 2001), lo cual hablaría de una mayor sensibilidad a los temas éticos, siendo más susceptibles a las capacitaciones. Si bien estos resultados no son concluyentes, esperan ser un aporte a la discusión teórica sobre la materia (Alatas, Cameron, Chaudhuri, Erkal \& Gangadharan, 2009; Dawson, 1995; Evans \& Palermo, 2005) y a los actuales esfuerzos internacionales por avanzar en el desafío de vincular ética, corrupción y género (Transparency International, 2020; United Nations Office on Drugs and Crime [UNODC], 2020).

\section{CONCLUSIONES}

Desde un punto de vista práctico, el presente estudio exploratorio ha permitido conocer los efectos que el módulo "Ética Pública, Probidad y Transparencia" tiene en las personas que participan del curso Inducción a la Administración del Estado impartido por el CEA de la CGR.

Desde un punto de vista teórico, es necesario reconocer algunas limitaciones que esta investigación presenta. La primera se refiere al limitado número de participantes analizados, cuestión que creemos importante para futuras investigaciones. Lo segundo se refiere a que no se incorporaron otros factores en el estudio como el tipo de instructor que realiza las capacitaciones, y las formas de desarrollar las capacitaciones, que pudiesen también tener un impacto en los resultados.

A pesar de lo anterior, se han establecido una serie de conclusiones relevantes para el estudio de las capacitaciones sobre integridad en el sector público. En primer lugar, creemos que la investigación muestra la utilidad que las capacitaciones tienen en el perfeccionamiento de los marcos éticos de los y las participantes. En términos generales, el grupo participante demostró tener una disposición a considerar éticamente menos aceptable los casos presentados luego de la capacitación; lo que permitiría concluir que las personas mejoran la alineación de sus valores individuales con los valores de la función pública y se vuelven más críticas con aquellas acciones que podrían violar la integridad institucional. El desafío en esta línea es evaluar si este cambio en la percepción ética tiene un efecto solo en el corto plazo o, por el contrario, al volver a su puesto de trabajo, el personal sostiene en el mediano y largo plazo este marco ético que la capacitación ha ajustado (Van Montfort et al., 2013).

Un segundo aporte a la discusión teórica, se refiere a los efectos diferenciados entre tipos de funcionarios. Creemos que es relevante reconocer que las capacitaciones en ética influyen de distinta forma a funcionarios con distinta antigüedad en el sector público y por género. Por otro lado, la focalización con que se estructura la metodología de una actividad de capacitación ética parece ser un elemento relevante para evaluar la efectividad de estas intervenciones. Tal como se señaló inicialmente en la revisión teórica realizada y a partir de Cooper (2006), las capacitaciones resultan más útiles si se entienden como instancias de reflexión frente a determinadas situaciones éticas específicas. Esto ha sido reafirmado por los resultados obtenidos, donde se identificaron modificaciones en los marcos éticos de aquellas situaciones que fueron abordadas de manera detallada, detenida y con un análisis de contexto relevante. Queda pendiente para otras investigaciones, si el efecto detectado perdura en el tiempo (Warren, Gaspar \& Laufer, 2014).

La evidencia teórica y las conclusiones alcanzadas en esta investigación permiten identificar recomendaciones prácticas para perfeccionar el efecto que tienen las capacitaciones éticas. Así, es 
RAP | Efecto de las capacitaciones en la reflexión sobre ética y corrupción

parece indispensable avanzar en dos líneas concretas. Por un lado, a través del diseño e implementación de actividades con metodologías reflexivas sobre instancias sencillas y cotidianas para el grupo de capacitado y, por otro lado, mediante la utilización de situaciones y casos éticos diferenciados según el tipo de participante al que se dirige la capacitación. Los resultados alcanzados permiten establecer que estos son factores fundamentales para aumentar la efectividad de las capacitaciones éticas en nuestras organizaciones. 


\section{REFERENCIAS}

Alatas, V., Cameron, L., Chaudhuri, A., Erkal, N., \& Gangadharan, L. (2009). Gender, culture, and corruption: Insights from an experimental analysis. Southern Economic Journal, 75(3), 663-680.

Beeri, I., Dayan, R., Vigoda-Gadot, E., \& Werner, S. B. (2013). Advancing Ethics in Public Organizations: The Impact of an Ethics Program on Employees' Perceptions and Behaviors in a Regional Council. Journal of Business Ethics, 112(1), 59-78. Recuperado de https://doi.org/10.1007/s10551-012-1232-7

Bermúdez, J. (2017). El anillo de giges, ¿Podemos realmente ganar la lucha contra la corrupción? Santiago, Chile: Contraloría General de la República de Chile.

Boyne, G. A. (2002). Public and private management: what's the difference? Journal of Management Studies, 39(1), 97-122.

Cochrane, C. (2020). Teaching integrity in the public sector: Evaluating and reporting anti-corruption commissions' education function. Teaching Public Administration, 38(1), 78-94. Recuperado de https:// doi.org/10.1177/0144739419851147

Cooper, T. (2006). The Responsible Administrator: an approach to ethics for the administrative role (Jossey Bass Nonprofit \& Public Management Series, 5a ed.). San Francisco, CA: Jossey-Bass.

Dawson, L. M. (1995). Women and men, morality and ethics. Business Horizons, 38(4), 61-68. Recuperado de https://doi.org/10.1016/0007-6813(95)90010-1

Delaney, J. T., \& Sockell, D. (1992). Do company ethics training programs make a difference? An empirical analysis. Journal of Business Ethics, 11(9), 719-727.

Dirección Chile Compra. (2018). Chile, Evaluación del sistema de compras públicas: Metodología MAPS. Santiago, Chile: Autor.

Dwivedi, O. P., \& Engelbert, E. A. (1981). Education and Training for Values and Ethics in the Public Service: An International Perspective. Public Personnel Management, 10(1), 140-145. Recuperado de https://doi.org/10.1177/009102608101000120

Evans, A., \& Palermo, J. (2005). Gender \& Ethics. Law Institute Journal, 10(3), 40-45.
Frisque, D. A., \& Kolb, J. A. (2008). The effects of an ethics training program on attitude, knowledge, and transfer of training of office professionals: A treatment- and control-group design. Human Resource Development Quarterly, 19(1), 35-53.

Gomes, N. F. (2014). Ética en la administración pública: desafíos y oportunidades. Revista de Administração Pública, 48(4), 1029-1050.

Hejka-Ekins, A. (2001). Ethics in in-service training. In T. L. Cooper (Ed.), Handbook of Administrative Ethics (2 ed.). London, UK: Routledge.

Huberts, L., \& Hoekstra, A. (Eds.). (2016). Integrity Management in The Public Sector - The Dutch Approach. The Hague, The Netherlands: Dutch National Integrity Office.

Inter-American Development Bank. (2008). Evaluación del Sistema Nacional de Compras y Contrataciones Públicas. Santiago, Chile: Autor.

Jiménez Asensio, R. (2017). Cómo prevenir la corrupción: integridad y transparencia. Madrid, España: La Catarata.

Kaptein, M. (2009). Ethics programs and Ethical culture: A next step in unraveling their multi-faceted relationship. Journal of Business Ethics, 89(2), 261281. Recuperado de https://doi.org/10.1007/s10551008-9998-3

Kaptein, M. (2015). The Effectiveness of Ethics Programs: The Role of Scope, Composition, and Sequence. Journal of Business Ethics, 132(2), 415-431. Recuperado de https://doi.org/10.1007/s10551-0142296-3

Kirkpatrick, D., \& Kirkpatrick, J. (2006). Evaluating training programs: The four levels. Oakland, CA: Berrett-Koehler Publishers.

Lara A., J. L. (2017). Funcionamiento del sistema nacional de compras públicas y evaluación del caso del Tribunal de Contratación Pública: propuestas de cambio. In I. Aninat, \& S. Razmilic (Eds.), Un Estado para la Ciudadania (pp. 715-752). Santiago, Chile: Centro de Estudios Públicos.

Lewis, C. W., \& Gilman, S. C. (2012). The Ethics Challenge in Public Service: A Problem-Solving Guide. Hoboken, NJ: John Wiley \& Sons.

Maesschalck, J. (2004). Approaches to ethics management in the public sector: A proposed 
extension of the compliance-integrity continuum. Public Integrity, 7(1), 20-41.

Medeiros, K. E., Watts, L. L., Mulhearn, T. J., Steele, L. M., Mumford, M. D., \& Connelly, S. (2017). What is Working, What is Not, and What We Need to Know: a Meta-Analytic Review of Business Ethics Instruction. Journal of Academic Ethics, 15(3), 245-275. Recuperado de https://doi.org/10.1007/ s10805-017-9281-2

Menzel, D. C. (2001). Ethics Management in Public Organizations: What, Why, and How? In T. L. Cooper (Ed.), Handbook of Administrative Ethics (pp. 355-366). New York, N.Y: Marcel Dekker Inc.

Menzel, D. C. (2007). Ethics management for public administrators: Building organizations of integrity. New York, NY: M.E. Sharpe.

Menzel, D. C. (2015). Research on Ethics and Integrity in Public Administration: Moving Forward, Looking Back. Public Integrity, 17(4), 343-370. Recuperado de https://doi.org/10.1080/10999922. 2015.1060824

Meyer, A. D., \& Fath Meyer, J. M. (2012). Evaluación de las contrataciones públicas en Chile (Reforma del Estado II), Santiado, Chile: Centro de Estudios Públicos.

Neu, D., Everett, J., \& Rahaman, A. S. (2015). Preventing corruption within government procurement: Constructing the disciplined and ethical subject. Critical Perspectives on Accounting, 28, 49-61. Recuperado de https://doi.org/10.1016/j. cpa.2014.03.012

Organisation for Economic Co-operation and Development. (2020). OECD Public Integrity Handbook. Paris, France: Autor.

Osorio, V., \& Rodríguez, M.del P. (2018). Modelo para la Evaluación de Programas de Ética Organizacional en Universidades. Información Tecnológica, 29(1), 59-70. Recuperado de https://doi.org/10.4067/ s0718-07642018000100059

Pallai, K., \& Gregor, A. (2016). Assessment of effectiveness of public integrity training workshops for civil servants - A case study. Teaching Public Administration, 34(3), 247-269. Recuperado de https://doi.org/10.1177/0144739416650431
Park, H., \& Blenkinsopp, J. (2013). The impact of ethics programmes and ethical culture on misconduct in public service organizations. International Journal of Public Sector Management, 26(7), 520533. Recuperado de https://doi.org/10.1108/ IJPSM-01-2012-0004

Pliscoff, C. (2019). Ethics and Public Administration in Latin America. In A. Farazmand (Ed.), Global Encyclopedia of Public Administration, Public Policy, and Governance (pp. 1-12). New York, NY: Springer. Recuperado de https://doi.org/10.1007/978-3-31931816-5_2675-2

Ritter, B. A. (2006). Can business ethics be trained? A study of the ethical decision-making process in business students. Journal of Business Ethics, 68(2), 153-164. Recuperado de https://doi.org/10.1007/ s10551-006-9062-0

Roberts, R. (2009). The rise of compliance-based ethics management: Implications for organizational ethics. Public Integrity, 11(3), 261-278.

Rohr, J. A. (1989). Ethics for bureaucrats: An essay on law and virtue (2a Ed.). New York, NY: Marcel Dekker Inc.

Steele, L. M., Mulhearn, T. J., Medeiros, K. E., Watts, L. L., Connelly, S., \& Mumford, M. D. (2016). How Do We Know What Works? A Review and Critique of Current Practices in Ethics Training Evaluation. Accountability in Research, 23(6), 319350. Recuperado de https://doi.org/10.1080/08989 621.2016 .1186547

Swamy, A., Knack, S., Lee, Y., \& Azfar, O. (2001). Gender and corruption. Journal of Development Economics, 64(1), 25-55.

Thompson, B. L. (1990). Ethics Training Enters the Real World. Training, 27(10), 82-94.

Toro, J., \& Rodríguez, M. D. P. (2017). Formación en ética en las organizaciones: Revisión de la literatura. Informacion Tecnologica, 28(2), 167-180. Recuperado de https://doi.org/10.4067/S071807642017000200018

Transparency International. (2020). Gender. Recuperado de https://www.transparency.org/en/ our-priorities/gender-and-corruption

Trevino, L. K., \& Brown, M. E. (2004). Managing to be ethical: Debunking five business ethics myths. Academy of Management Perspectives, 18(2), 69-81. 
Tuana, N. (2007). Conceptualizing moral literacy. Journal of Educational Administration, 45(4), 364-378. Recuperado de https://doi. org/10.1108/09578230710762409

United Nations Office on Drugs and Crime. (2020). Gender \& Corruption. Recuperado de https://www. unodc.org/unodc/gender/gender-and-corruption. html

Van Montfort, A., Beck, L., \& Twijnstra, A. (2013). Can integrity be taught in public organizations? The effectiveness of integrity-training programs for municipal officials. Public Integrity, 15(2), 117-132.

Warren, D. E., Gaspar, J., \& Laufer, W. S. (2014). Is Formal Ethics Training Merely Cosmetic?: A
Study of Ethics Training and Ethical Organizational Culture. Business Ethics Quarterly, 24(1), 85-117.

Worthley, J. A. (1981). Ethics and Public Management: Education and Training. Public Personnel Management, 10(1), 41-47. Recuperado de https://doi.org/10.1177/009102608101000106

Worthley, J. A., \& Grumet, B. R. (1983). Ethics and Public Administration: Teaching What "Can't be Taught." The American Review of Public Administration, 17(1), 54-67. Recuperado de https:// doi.org/10.1177/027507408301700106

Yizraeli, D., \& Shilo, L. (2001). The effectiveness of ethics programs in organizations. Human Resources, 165-166, 14-15.

\section{Cristian Pliscoff-Varas}

https://orcid.org/0000-0002-6918-4587

Ph.D. Public Administration en University of Southern California; Administrador Público en la Universidad de Chile; Profesor Asociado en la Universidad de Chile. E-mail: cpliscof@iap.uchile.cl

\section{Nicolás Lagos-Machuca}

https://orcid.org/0000-0001-9140-1958

Master en Gobierno y Gerencia Pública en la Universidad de Chile; Administrador Público en la Universidad de Chile; Analista Unidad de Estudios en el Gabinete Contralor de la Contraloría General de la República de Chile. E-mail: nmlagos@gmail.com 


\section{ANEXOS}

TABLA 1 PUNTAJES PROMEDIOS PARA CADA SITUACIÓN ANTES Y DESPUÉS DE LA CAPACITACIÓN Y
DIFERENCIA ENTRE AMBAS EVALUACIONES

\begin{tabular}{|c|c|c|c|c|c|}
\hline \multirow{2}{*}{\multicolumn{2}{|c|}{ Situación }} & \multicolumn{2}{|c|}{ Antes } & \multicolumn{2}{|c|}{ Después } \\
\hline & & Media & D.Est & Media & D.Est \\
\hline 1 & Utilizar la impresora institucional para fines personales. & 4,70 & 1,79 & 5,10 & 2,05 \\
\hline 2 & Llegar tarde al trabajo de manera frecuente. & 6,07 & 1,81 & 6,63 & 1,54 \\
\hline 3 & No cumplir con los plazos laborales comprometidos. & 5,96 & 1,45 & 6,77 & 1,39 \\
\hline 4 & $\begin{array}{l}\text { Marcar el control horario y luego ir a realizar trámites } \\
\text { personales. }\end{array}$ & 6,63 & 1,65 & 7,47 & 1,15 \\
\hline 5 & $\begin{array}{l}\text { Informar al encargado de un proceso de contratación de } \\
\text { personal la postulación de un amigo/familiar para que sea } \\
\text { considerado en el concurso. }\end{array}$ & 5,90 & 2,09 & 6,74 & 1,69 \\
\hline 6 & $\begin{array}{l}\text { Alterar el orden de atención de solicitudes para priorizar la de } \\
\text { un conocido. }\end{array}$ & 6,67 & 1,71 & 7,20 & 1,19 \\
\hline 7 & $\begin{array}{l}\text { Agilizar el trámite de un amigo/familiar/colega a quien le debías } \\
\text { un favor. }\end{array}$ & 6,81 & 1,49 & 7,14 & 1,35 \\
\hline 8 & $\begin{array}{l}\text { Distribuir desproporcionadamente el trabajo entre personas con } \\
\text { la misma responsabilidad. }\end{array}$ & 6,41 & 1,64 & 6,89 & 1,66 \\
\hline 9 & $\begin{array}{l}\text { Modificar la información de documentos de trabajo para obtener } \\
\text { un resultado deseado pero irreal. }\end{array}$ & 7,69 & 1,56 & 8,09 & 1,28 \\
\hline 10 & $\begin{array}{l}\text { Utilizar el correo institucional para intentar obtener un beneficio } \\
\text { personal. }\end{array}$ & 6,67 & 1,71 & 7,04 & 1,66 \\
\hline 11 & $\begin{array}{l}\text { Entregar información confidencial de la institución a amigos y/0 } \\
\text { familiares. }\end{array}$ & 7,69 & 1,61 & 7,86 & 1,47 \\
\hline 12 & $\begin{array}{l}\text { Presentar una licencia médica por enfermedad sin estarlo } \\
\text { realmente. }\end{array}$ & 7,13 & 1,56 & 7,68 & 1,48 \\
\hline 13 & $\begin{array}{l}\text { Informar al encargado de un proceso de licitación de la } \\
\text { postulación de un proveedor de un amigo/familiar para que sea } \\
\text { considerado en el concurso. }\end{array}$ & 7,29 & 1,69 & 7,30 & 1,52 \\
\hline 14 & Aceptar un regalo de un proveedor de la institución. & 6,44 & 1,99 & 6,51 & 1,84 \\
\hline 15 & $\begin{array}{l}\text { Solicitar un regalo a un usuario de la entidad a cambio de } \\
\text { agilizar un trámite. }\end{array}$ & 7,78 & 1,51 & 7,78 & 1,57 \\
\hline
\end{tabular}

Fuente: Elaborada por los autores. 
RAP | Efecto de las capacitaciones en la reflexión sobre ética y corrupción

\section{TABLA 2 COEFICIENTES DE CORRELACIÓN R² PARA PUNTAJES PRE Y POST CAPACITACIÓN}

\begin{tabular}{|c|c|c|c|c|c|c|}
\hline ACCIÓN & Género (PRE) & Género (POST) & Edad (PRE) & Edad (POST) & Años AP (PRE) & Años AP (POST) \\
\hline 1 & $-0,018$ & 0,284 & 0,088 & 0,115 & 0,083 & $-0,005$ \\
\hline 2 & $-0,076$ & 0,120 & 0,053 & 0,193 & 0,038 & 0,047 \\
\hline 3 & 0,134 & 0,296 & 0,056 & 0,104 & $-0,113$ & $-0,141$ \\
\hline 4 & 0,010 & 0,193 & 0,037 & 0,052 & $-0,074$ & $-0,160$ \\
\hline 5 & $-0,206$ & 0,368 & 0,048 & 0,074 & $-0,055$ & 0,007 \\
\hline 6 & $-0,046$ & 0,311 & $-0,067$ & $-0,137$ & $-0,158$ & $-0,294$ \\
\hline 7 & 0,046 & 0,333 & $-0,132$ & $-0,021$ & $-0,100$ & $-0,146$ \\
\hline 8 & 0,132 & 0,308 & $-0,049$ & 0,031 & $-0,081$ & $-0,153$ \\
\hline 9 & 0,002 & 0,285 & 0,079 & $-0,040$ & $-0,041$ & $-0,040$ \\
\hline 10 & 0,089 & 0,394 & $-0,008$ & 0,066 & $-0,166$ & $-0,204$ \\
\hline 11 & $-0,030$ & 0,286 & $-0,031$ & $-0,017$ & $-0,095$ & $-0,181$ \\
\hline 12 & $-0,025$ & 0,247 & 0,098 & 0,175 & $-0,058$ & 0,022 \\
\hline 13 & $-0,022$ & 0,344 & 0,139 & 0,097 & 0,059 & 0,037 \\
\hline 14 & 0,028 & 0,265 & 0,062 & 0,110 & $-0,053$ & $-0,146$ \\
\hline 15 & 0,048 & 0,292 & $-0,035$ & $-0,042$ & $-0,072$ & $-0,186$ \\
\hline
\end{tabular}

Fuente: Elaborada por los autores. 